\title{
Mesoscopic Resistance Fluctuations in Cobalt Nanoparticles
}

\author{
Y. G. Wei, X. Y. Liu, L. Y. Zhang, and D. Davidović \\ Georgia Institute of Technology, Atlanta, GA 30332
}

(Dated: May 30, 2018)

\begin{abstract}
We present measurements of mesoscopic resistance fluctuations in cobalt nanoparticles and study how the fluctuations with bias voltage, bias fingerprints, respond to magnetization reversal processes. Bias fingerprints rearrange when domains are nucleated or annihilated. The domain-wall causes an electron wavefunction phase-shift of $\approx 5 \pi$. The phase-shift is not caused by the Aharonov-Bohm effect; we explain how it arises from the mistracking effect, where electron spins lag in orientation with respect to the moments inside the domain-wall. Dephasing time in Co at $0.03 K$ is short, $\tau_{\phi} \sim p s$, which we attribute to the strong magnetocrystalline anisotropy.
\end{abstract}

In micron scale metallic samples at low temperatures, interference among scattered electron waves creates noticeable contributions to sample resistance, including random but reproducible fluctuations in conductance (CF). 1, 2, 3] One remarkable consequence is that the resistance of phase-coherent samples becomes sensitive to microscopic impurity configurations. In this work we investigate the resistance of mesoscopic ferromagnets at low temperatures and find a similar result that the resistance is very sensitive to the magnetic state of the sample. In particular, we observe significant wave-function phase-shifts generated by domain-walls.

Mesoscopic effects in ferromagnets could be significantly different from mesoscopic effects in normal metals. 4, 5, 6, 7] While normal metals with a short mean-free-path do not exhibit classical magnetoresistance (MR), weakly disordered ferromagnets with a similar mean-free-path display MR, which includes domainwall resistance (DWR) 8, 9, 10, 11, 12, 13, 14, 15, 16, 17, 18] and anisotropic magnetoresistance (AMR) 19. MR could lead to novel mesoscopic effects because the wavefunction-phase depends on the scattering potential. [6, 7]

Signatures of mesoscopic electron transport in ferromagnets have been reported. 20, 21, 22, 23] However, the dependence of the phase of the electron wavefunction on magnetization reversal processes have not been measured yet. A representative sample is shown in Fig. 1-A. A cobalt (Co) particle of $200 \mathrm{~nm}$ diameter and $10 \mathrm{~nm}$ thickness is in electric contact with two copper $(\mathrm{Cu})$ leads of $50 \mathrm{~nm}$ thickness. The gap between $\mathrm{Cu}$ leads is $100 \mathrm{~nm}$. The device cross-section is displayed in Fig. 1 1 B.

Samples are made by electron beam lithography and shadow metal deposition. First, a Co nanoparticle is deposited at $10^{-7}$ Torr base pressure and a rate of $0.5 \mathrm{~nm} / \mathrm{s}$. Deposition is stopped when the Co thickness $(t)$ reaches $10 \mathrm{~nm}$. Then we rotate the sample without breaking the vacuum and deposit $\mathrm{Cu}$ at $0.5 \mathrm{~nm} / \mathrm{sec}$. The interface between $\mathrm{Co}$ and $\mathrm{Cu}$ is nearly free from adsorbates because the Co surface is exposed to base pressure for less than 10 seconds. The nanoparticle is completely isolated from any other ferromagnet in a vicinity of $1.5 \mu \mathrm{m}$ to remove the influence of stray magnetic field from other magnetic

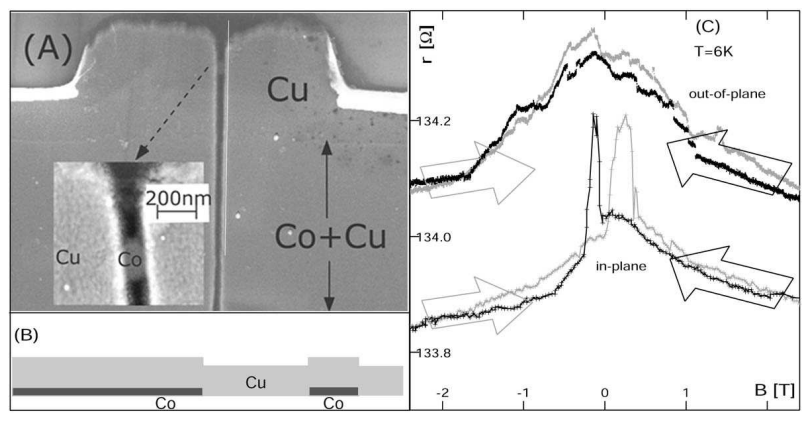

FIG. 1: A: Image of a typical sample. B: Sketch of the device cross-section taken along the white line in Fig. 1-A. C: Outof-plane and in-plane MR.

parts of the device.

The sample is next exposed to air and transferred to a dilution refrigerator. Surface oxidation reduces the metal thickness and covers the film with a cobalt-oxide $(\mathrm{CoO})$ layer. The time of air exposure before evacuation in the dilution refrigerator is less than one hour. We measure the resistance of Co films with various thicknesses in situ and monitor how the resistance increases when the films are exposed to air for one hour. From this we infer that the thickness of Co-metal is reduced by approximately $2 \mathrm{~nm}$ after one hour of air exposure. The sheet resistance of the Co film at $4.2 \mathrm{~K}$ temperature is $R_{S} \approx 120 \Omega$.

$\mathrm{CoO}$ is antiferromagnetic and the $\mathrm{Co} / \mathrm{CoO}$ interface generates an exchange-bias effect in Co, 17, 24, 25] which leads to pinning of the magnetization and the enhancement of the coercive field. We expect that domainwalls are nucleated at the interface between the exposed $\mathrm{Co}$ and unexposed $\mathrm{Co}$ (under $\mathrm{Cu}$ ) by applying well defined magnetic fields, analogous to the similar behavior shown in Ref. 17]. The nanoparticle can support domains because the domain-wall width $\left(\delta_{w}\right)$ in Co $\left(\delta_{w}=15 \mathrm{~nm}[9,10,11]\right)$ is much smaller than the diameter.

Four samples were studied at low temperatures, and the reproducible main result is that the correlation field is strongly suppressed near zero field. The dephasing time is also reproducible among samples. 
Differential resistance $(r=d V / d I)$ is measured as a function of DC-bias voltage $(V)$ and the applied magnetic field $(\mathrm{B}),(r(V, B))$. The applied current is $I+i \cos (2 \pi f t)$, where $i=0.5 \mu \mathrm{A}$, and $f=80 \mathrm{~Hz}$. Then, $r$ is obtained by measuring the AC-voltage across the sample with a lock-in amplifier. At $0.03 K$, we confirm that $r(V, B)$ is independent of $i$ when $i<0.5 \mu \mathrm{A}$. The resistance of $\mathrm{Cu}$ leads is about $10 \Omega$ and is not subtracted from $r$.

The out-of-plane (OP) and the in-plane (IP, magnetic field perpendicular to the current) $\mathrm{MR}$ at $T=6 \mathrm{~K}$ are shown in Fig. 1.B. First we discuss the IP-MR data. The magnetic field is initially set to $-12 \mathrm{~T}$, then it is reduced to $-2.4 \mathrm{~T}$, and then it is cycled between $-2.4 \mathrm{~T}$ and $2.4 \mathrm{~T}$.

The IP-MR graph has hysteresis. There are two sharp resistance transitions in each field direction. The low and the high field transitions indicate nucleation and annihilation of domains, respectively. The smaller coercive fields are symmetric, $B_{C}= \pm 62 m T$, which can be explained if the magnetization is first reversed in unexposed $\mathrm{Co}$ (under $\mathrm{Cu}$ ), as expected. The larger coercive fields are $330 m T$ and $-220 m T$. The magnetic moments in exchange biased Co change direction at these coercive fields. The coercive field is larger in magnitude when $B$ increases, because $B$ is initially $-12 T$.

The resistance increases when the domains are nucleated. The increase is explained by the AMR inside the wall, which arises from the dependence of the conduction electron scattering rate on the angle between the current and the magnetization. 19

Next we discuss the OP-MR in Fig. 1-B. OP-MR exhibits a broad maximum at $B=0$ and a weak hysteresis. The maximum is explained as arising from the rotation of magnetic moments supported by the shape anisotropy. As the magnetic moments rotate into the film plane, the angle between magnetic moments and the current is reduced, so $r$ increases.

Extracting CF's: We describe the analysis of the OPfield data. The analysis of the IP-field data is equivalent. The resistance increases by $\approx 6 \Omega$ when the temperature drops from $6 K$ to $0.03 K$. Similar effect is reported in Co films at temperatures above $1.5 K$ [26] and is attributed to enhancement of electron-electron interactions caused by phase-coherence. 27]

To study mesoscopic effects, we obtain the dependence of $r$ on two independent parameters, $V$ and $B(r(V, B))$. The dependence is obtained by quickly sweeping the bias voltage, while the applied field is slowly changing. Fig. 2. A displays $r(V, B)$ when $B$ is OP. The cross in this image displays maximums in resistance versus field and voltage, centered at zero field and voltage, respectively.

The average resistance versus field and voltage are defined as $r_{0}(B)=\int_{-V_{\max }}^{V_{\max }} r(V, B) d V / 2 V_{\max }$ and $r_{0}(V)=$ $\int_{-B_{\max }}^{B_{\max }} r(V, B) d B / 2 B_{\max }$, respectively, where $B_{\max }=$ $12 T$, and $V_{\max }=4.2 \mathrm{mV}$. The averages are shown in Figs. 2-B and C. The resistance averaged over both $V$

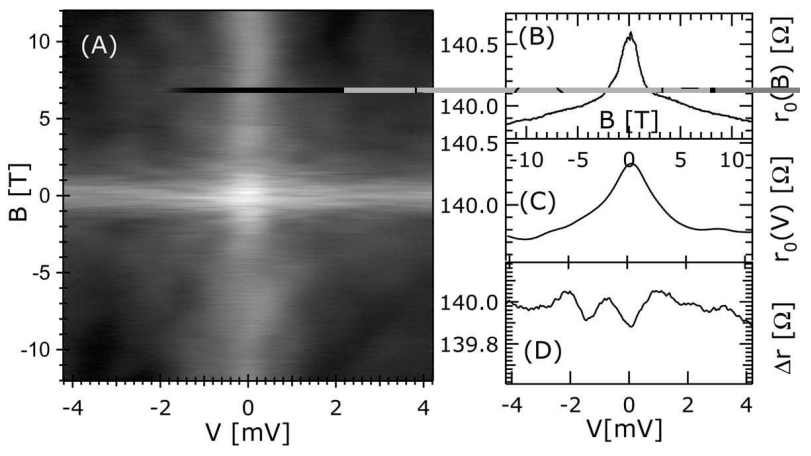

FIG. 2: A: Differential resistance $(r)$ versus bias voltage and out-of-plane magnetic field at $0.03 \mathrm{~K}$. B and C: Average resistance versus out-of-plane field and bias voltage, respectively, defined in text. D: Fluctuations in resistance with bias voltage, $r(V, B)-r_{0}(V)+r_{0}$, at $B=-5.5 T$.

and $B$ is $r_{0}=140 \Omega$.

The average MR (Fig. 2-B) at $0.03 \mathrm{~K}$ is enhanced compared to the AMR at $6 \mathrm{~K}$. This enhancement suggests that weak localization effect contributes to MR at low temperatures. Prior research in Co-films did not find any weak localization effects at temperatures above $1.5 \mathrm{~K}$. 26. Our temperatures are much lower than $1.5 \mathrm{~K}$, which could explain the difference between the results.

The weak localization MR contribution cannot be extracted from data because of the internal field of Co. The total magnetic field acting on conduction electrons in Co cannot be equal to zero; it is equal to the sum of the applied field and the internal field $(1.8 \mathrm{~T})$, which is much larger than the coercive fields. Thus, the low-field contributions to quantum interference effects, such as weak antilocalization, are experimentally inaccessible. In the remainder of the text, we study $\mathrm{CF}$ only.

The resistance maximum with voltage in Fig. 2. $\mathrm{C}$ is a consequence of the electron-electron interaction enhancement effect (EE) in mesoscopic samples. 28 Resistance fluctuations in Fig. 2-A are superimposed with the EEeffect [28] and the AMR. To better display the fluctuations in resistance with voltage, we find the difference between $r(V, B)$ and the average EE-effect in Fig. 2. C. The resulting resistance as a function of voltage at fixed field is shown in Fig. 2-D. The resistance now clearly exhibits fluctuations with voltage. The fluctuations are reproducible and are found at low temperatures only.

The fluctuations in $r$ with $V$ represent changes in electron interference from constructive to destructive as a function of electron energy. 1] Root-mean-square (rms) of the fluctuations is $0.1 \Omega$, which corresponds to rms-CF of $0.05 e^{2} / h$. The fluctuation amplitude is $\ll e^{2} / h$, showing that the dephasing time must be much shorter than the transport time. The rms does not change with $V$, showing that the heating effects are weak.

The correlation voltage $V_{C}$ is given by the average spacing between minima and maxima, $V_{C} \sim 0.5 \mathrm{mV}$. The 


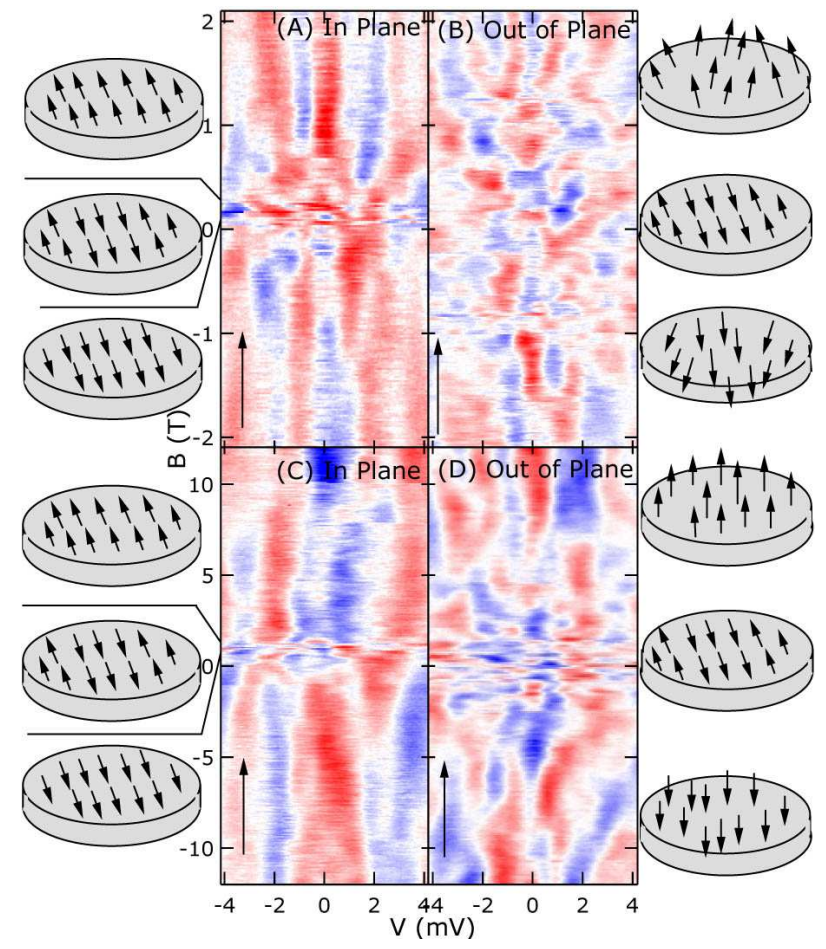

FIG. 3: A and B: Fluctuations in differential resistance, $r(V, B)-r_{0}(V)-r_{0}(B)$, with $V$ and the IP-field and the OP-field, respectively. $\mathrm{C}$ and $\mathrm{D}$ : same as $\mathrm{A}$ and $\mathrm{B}$, but in a wider field range. The minima and the maxima of differential resistance with $B$ and $V$ correspond to constructive and destructive electron interference, as described in text. The schematics indicate the expected magnetic configurations.

meaning of $V_{C}$ is that changing the electron energy by $e V_{C}$ changes the electron-phase by $\pi$. $V_{C}$ is related to the dephasing time $\tau_{\phi}$ as $\left.|e| V_{C}=\hbar / \tau_{\phi}, 29\right]$ so $\tau_{\phi}=1.3 p s$.

Discussion of the results: To display the fluctuating part of the resistance, we subtract the average EE-effect and MR from the resistance. Four red-white-blue images in Fig. 3 display CFs with magnetic field and bias voltage at $0.03 \mathrm{~K}$ refrigerator temperature. Red and blue regions indicate larger and smaller resistance, respectively.

The fluctuations with field and voltage, represented in Fig. 3] are not reproducible when the field is varied arbitrarily. However, the fluctuations are reproducible when $B$ varies between two fields in the same direction after an initial training with one field cycle.

In Figs. 3-A and B, there is a noticeable difference between dependence of the bias-fingerprints on the IP and the OP-fields. If the IP-field varies from $-2 \mathrm{~T}$ to 0 , the resistance maxima and minima with voltage shift weakly. By contrast, when $B$ is changing OP, bias-fingerprints shift or rearrange several times. These rearrangements are indicated by the appearances and disappearances of the red and blue regions in Fig. 3.B.

Fig. 3. $\mathrm{C}$ shows that bias fingerprints vary weakly with the IP-field in the field range $-12 T<B<0$. But, when the IP-field changes sign and reaches the coercive field, the bias-fingerprints rearrange at the coercive field. This shows that the domain-walls generate significant electron-phase shifts, at least on the order of $\pi$.

Before discussing the physical origin of the rearrangements in bias-fingerprints, we analyze the strong field data in Figs. 3 . C and D, $2 T<|B|<12 T$. Comparing Fig. 3. C and Fig. 3.D in this field-range, we observe that bias fingerprints vary faster with the OP-field. Alternatively, the characteristic field scale, which rearranges the bias-fingerprint in strong field, is smaller in the OPdirection. The correlation field $B_{C}$ is the average spacing between the red and the blue regions along $B$ axes; $B_{C} \approx 4 T$ and it is weakly dependent on $V$, confirming that the heating is not significant.

In a strong OP-field, magnetization is saturated; and $B_{C}$ is given by the field for a flux quantum over the phase coherent area, $\Phi_{0} / L_{\phi}^{2}$, where $\Phi_{0}=h / e$ is the flux quantum, and $L_{\phi}$ is the dephasing length. We find $L_{\phi} \approx 30 \mathrm{~nm}$. Assuming a mean-free-path $l=5 \mathrm{~nm}$ and the Fermi velocity $v_{F}=1.4 \cdot 10^{6} \mathrm{~m} / \mathrm{s}$, the electron dephasing time is $L_{\phi}^{2} /\left(v_{F} l / 3\right)=0.4 p s$, in agreement with $\tau_{\phi}$ obtained before, within an order of magnitude. In the IP-direction, $B_{C}$ is larger; because the phase coherent area perpendicular to the field is smaller, $B_{C}=\Phi_{0} / t L_{\phi} \approx 12 T$.

Now we discuss the rearrangements in bias-fingerprints at the coercive fields, Fig. B. A and C. The internal field switches at the coercive fields. In Co, the internal field change is less than 3.6T, much smaller than the IP-field for a flux quantum (12T). So the Aharonov-Bohm effect cannot be responsible for the rearrangements.

Fig. 3. D shows that the density of red and blue regions increases when $|B|<1.5 T$. In Fig. B3.B, there are about five red and blue regions along B-axes between 0 and $1.5 \mathrm{~T}$. This shows that the magnetization rotation from IP to OP direction creates a phase-shift along a typical phase coherent electron trajectory of about $5 \pi$. Since the total field (internal plus applied) changes by $<3.3 T$ in this applied field range and the OP-field for a flux quantum is $4 T$, five resistance minima and maxima cannot originate from the Aharonov-Bohm effect.

We show that the phase-shift could originate from a weak mistracking effect when conduction electron spins lag behind the magnetic moments in the domainwall. [9, 10, 11, 30] The conduction electron spin tracks the local exchange field well when the angular rotational period around the exchange field is much smaller than the time of flight across the wall, which is equivalent to a large value of the tracking parameter $\xi=2 E_{e x} \delta_{w} / h v_{F}$. Here, $E_{e x}$ is the exchange energy between conduction electron spins and the spins responsible for ferromagnetism. After a conduction electron traverses the wall, the angle between the exchange field direction and the conduction electron spin is $\theta=1 / \xi$.

Using $E_{e x}=1 \mathrm{eV}$ and $\delta_{w}=15 \mathrm{~nm}$, one obtains 
$\xi \approx 7.3$. [9] The angular deviation increases the effective potential energy of the conduction electron by $\Delta=E_{e x}(1-\cos (\theta)) \approx 9 \mathrm{meV}$. The increase in effective potential energy contributes to DWR. 9, 10, 11, 30 In mesoscopic transport, however, electrons interfere among trajectories with diffusion times shorter than $\tau_{\phi}$, which leads to a correction in sample resistance. The wavefunction attains a phase-shift from this effective potential of $\Delta \tau_{\phi} / \hbar \approx 4.8 \pi$. The phase-shift is reduced to zero when the magnetic moments become parallel with each other. So the bias-fingerprints should rearrange about 5 times when they rotate into the OP-direction.

The dephasing length of $L_{\phi}=30 \mathrm{~nm}$ is very short. In a separate experiment, we measured two Co nanowires of lengths $500 \mathrm{~nm}$ and $800 \mathrm{~nm}$ and width $100 \mathrm{~nm}$ at $T=$ $0.03 K$ (not shown). These nanowires displayed no conductance fluctuations, confirming that $L_{\phi} \ll 500 \mathrm{~nm}$.

The dephasing process in ferromagnets is not well understood. In permalloy, experiments suggest that two level systems are important sources of dephasing. 23. Theoretically, domain-walls were are found to reduce the dephasing length. [6] But, the dephasing time $\tau_{\phi}=$ $\hbar / e V_{C}$ in our samples is independent of $\mathrm{B}$, because $V_{C}$ does not vary significantly with $\mathrm{B} ; \tau_{\phi}$ in strong field, in a single domain state, is approximately the same as $\tau_{\phi}$ at $B=0$ when domains are present. This demonstrates that the domain-walls are not responsible for short $\tau_{\phi}$.

The phase of the wavefunction is extremely sensitive to the position/presence of domain-walls, as indicated by the rearrangement of bias fingerprints in Fig. 3 The absence of domain-wall contribution to dephasing suggests that the electron interaction with the wall must be elastic. This situation is analogous to the sensitivity of conductance fluctuation with respect to changes in the impurity configurations. [1, 2, 3] In a thin film mesoscopic sample, motion of an impurity by the Fermi wavelength rearranges CFs. Nevertheless, the impurities do not contribute to dephasing when electron scattering is elastic.

Kasai, et al., found very short $L_{\phi}$ in $\mathrm{Ni}, L_{\phi} \approx$ $80 \mathrm{~nm}$. 22] Small $L_{\phi}$ is correlated with the large magnetocrystalline anisotropy in $\mathrm{Ni}$; the dephasing length in permalloy, which has negligible magnetocrystalline anisotropy, is $500 \mathrm{~nm}$. Since the magnetocrystalline anisotropy in Co is stronger than that in $\mathrm{Ni}$, the dephasing length of $30 \mathrm{~nm}$ in Co agrees with the trend that $L_{\phi}$ decreases with magnetocrystalline anisotropy. 22]

In conclusion, we demonstrate mesoscopic resistance fluctuations induced by the magnetization-reversal process in a Co nanoparticle. The fluctuations are explained by the spin mistracking effect in electron transport through domain-walls. The dephasing length at low temperatures is only $30 \mathrm{~nm}$, which is attributed to the large magnetocrystalline anisotropy in $\mathrm{Co}$, in agreement with the trend established before, but not understood theoretically.
This work is performed in part at the Georgia Tech electron microscopy facility. We thank P. Brouwer and A. Zangwill for valuable discussions. his research is supported by the DOE grant DE-FG02-06ER46281 and the David and Lucile Packard Foundation grant No. 200013874 .

[1] S. Washburn and R. A. Webb, Rep. Prog. Phys. 55, 1311 (1992).

[2] P. A. Lee and A. D. Stone, Phys. Rev. Lett. 55, 1622 (1985).

[3] B. L. Altshuler and D. E. Khmelnitskii, Jetp Lett. 42, 359 (1985).

[4] Y. Lyanda-Geller, I. L. Aleiner, and P. M. Goldbart, Phys. Rev. Lett 81, 3215 (1998).

[5] D. Loss, H. Schoeller, and P. M. Goldbart, Phys. Rev. B 48, 15218 (1993).

[6] G. Tatara and H. Fukuyama, Phys. Rev. Lett. 78, 3773 (1997).

[7] S. Adam, M. Kindermann, and P. W. Brouwer, condmat/0512287 (2006).

[8] A. D. Kent, J. Yu, and S. S. P. Parkin, J. Phys. Condens. Matter 13, R461 (2001).

[9] J. F. Gregg, W. Allen, K. Ounadjela, M. Viret, M. Hehn, S. M. Thompson, and J. M. D. Coey, Phys. Rev. Lett. 77, 1580 (1996).

[10] M. Viret, D. Vignoles, D. Cole, J. M. D. Coey, W. Allen, D. S. Daniel, and J. F. Gregg, Phys. Rev. B 53, 8464 (1996).

[11] U. Ebels, A. Radulescu, Y. Henry, L. Piraux, and K. Ounadjela, Phys. Rev. Lett. 84, 983 (2000).

[12] M. Viret, Y. Samson, P. Warin, A. Marty, F. Ott, E. Sondergard, O. Klein, and C. Fermon, Phys. Rev. Lett. 85, 3962 (2000).

[13] U. Ruediger, J. Yu, S. Zhang, A. D. Kent, and S. S. P. Parkin, Phys. Rev. Lett. 80, 5639 (1998).

[14] S. G. Kim, Y. Otani, K. Fukamichi, S. Yuasa, R. Nyvlt, and T. Katayama, IEEE Trans. Magn. 35, 2862 (1999).

[15] D. Ravelosona, A. Cebollada, F. Briones, C. DiazPaniagua, M. A. Hidalgo, and F. Batallan, Phys. Rev. B 59, 4322 (1999).

[16] R. Danneau, P. Warin, J. P. Attane, I. Petej, C. Beigne, C. Fermon, O. Klein, A. Marty, F. Ott, Y. Samson, et al., Phys. Rev. Lett. 88, 157201 (2002).

[17] D. Buntinx, S. Brems, A. Volodin, K. Temst, and C. VanHaesendonck, Phys. Rev. Lett. 94, 017204 (2005).

[18] C. Yu, S. F. Lee, J. L. Tsai, E. W. Huang, T. Y. Chen, Y. D. Yao, Y. Liou, and C. R. Chang, J. Appl. Phys. 93, 8761 (2003).

[19] T. R. Mcguire and R. I. Potter, IEEE Transactions on magnetics 11, 1018 (1975).

[20] K. Hong and N. Giordano, Phys. Rev. B 51, 9855 (1995).

[21] J. Aumentado and V. Chandrasekhar, Physica B 284288, 1742 (2000).

[22] S. Kasai, E. Saitoh, and H. Miyajima, Journal of applied physics 93, 8427 (2003).

[23] S. Lee, A. Trionfi, and D. Natelson, Phys. Rev. B 70, 212407 (2004).

[24] W. H. Meiklejohn and C. P. Bean, Phys. Rev. 102, 1413 (1956). 
[25] B. Hausmanns, T. P. Krome, and G. Dumpich, J. Appl. Phys. 93, 8097 (2003).

[26] M. Brands, A. Carl, and G. Dumpich, Europhysics Letters 68, 268 (2004).

[27] B. L. Altshuler and A. G. Aronov, in Electron-Electron Interactions in Disordered Systems, edited by A. L. Efros and M. Pollak (Elsevier and Amsterdam, 1985).
[28] K. E. Nagaev, Phys. Lett. A 189, 134 (1994).

[29] S. Datta, Electronic transport in mesoscopic systems (Cambridge University Press, 2002).

[30] P. M. Levy and S. F. Zhang, Phys. Rev. Lett. 79, 5110 (1997). 